\title{
Present and future role of FDG-PET/CT imaging in the management of gynecologic malignancies
}

\author{
Kazuhiro Kitajima • Yasuhiko Ebina • \\ Kazuro Sugimura
}

Received: 4 March 2014 / Accepted: 2 April 2014/Published online: 18 April 2014

(C) Japan Radiological Society 2014

\begin{abstract}
Integrated positron emission tomography/computed tomography (PET/CT) with 2-[18F]fluoro-2-deoxyD-glucose (FDG) is a useful technique for acquiring both glucose metabolic and anatomic imaging data using a single device in a single diagnostic session, and has opened up a new field of clinical oncologic imaging. FDG-PET/CT has been used successfully for the staging, treatment optimization, re-staging, therapy monitoring, and prognostication of uterine and ovarian cancers as well as various malignant tumors. The present review discusses the current role of FDG-PET/CT in the management of gynecologic malignancies, focusing on its usefulness and limitations for imaging such patients.
\end{abstract}

Keywords Fluorodeoxyglucose (FDG) · Positron emission tomography/computed tomography (PET/CT) . Cervical cancer - Endometrial cancer - Ovarian cancer

K. Kitajima $(\varangle) \cdot$ K. Sugimura

Department of Radiology, Kobe University Graduate School of Medicine, 7-5-2 Kusunoki-cho, Chuo-ku, Kobe 650-0017, Japan e-mail: kazu10041976@yahoo.co.jp;

kitajima@med.kobe-u.ac.jp

K. Sugimura

e-mail: sugimura@med.kobe-u.ac.jp

\section{Y. Ebina}

Department of Obstetrics and Gynecology, Kobe University Graduate School of Medicine, 7-5-2 Kusunoki-cho, Chuo-ku, Kobe 650-0017, Japan

e-mail: ebiyas@med.kobe-u.ac.jp

\section{Introduction}

In the late 1990s, positron emission tomography (PET) with 2-[18F]fluoro-2-deoxy-D-glucose (FDG), which exploits the increased utilization and high uptake of glucose by malignant cells, opened up a new field of clinical oncologic imaging. PET images intrinsically lack anatomic information, so precise localization of any suspicious lesions may be difficult. Recently, however, integrated positron emission tomography/computed tomography (PET/CT), in which a full-ring-detector clinical PET scanner and multidetector-row helical CT scanner are combined, has made it possible to acquire both metabolic and anatomic imaging data using a single device in a single diagnostic session, and this technique can allow precise anatomic localization of suspicious areas of increased FDG uptake. When used in a clinical setting, FDG-PET/CT leads to a significant improvement in diagnostic accuracy, and has had a considerable impact on patient management, including diagnosis, staging, treatment optimization, restaging, therapy monitoring, and prognostication of various malignant tumors. We herein review the current and future roles of FDG-PET/CT in the management of uterine and ovarian cancers, discussing its usefulness and limitations for imaging these patients.

\section{Differentiation between malignant and benign ovarian tumors}

At present, the diagnosis of primary ovarian cancer is based principally on gynecological examination, assay of specific serum tumor markers including CA125, and radiological imaging techniques such as transvaginal sonography, CT, and magnetic resonance imaging (MRI). Three groups have 
reported that PET/CT has relatively good diagnostic accuracy for differentiating between malignant and benign ovarian tumors: its sensitivity is $81-100 \%$; its specificity is $74-100 \%$; and its accuracy is $81-97 \%$ [1-5]. However, $\mathrm{PET} / \mathrm{CT}$ is not as efficient at diagnosing low-grade and early adenocarcinomas and borderline tumors with a small volume or low cellular density, and false-positive results have also been reported for endometrioma, mature cystic teratoma, and fibroma. The fact that FDG is a nonspecific radioactive tracer for ovarian cancer mmeans that diagnosing microscopic lesions is still a challenging task, even with the spatial resolution of currently available PET/CT systems.
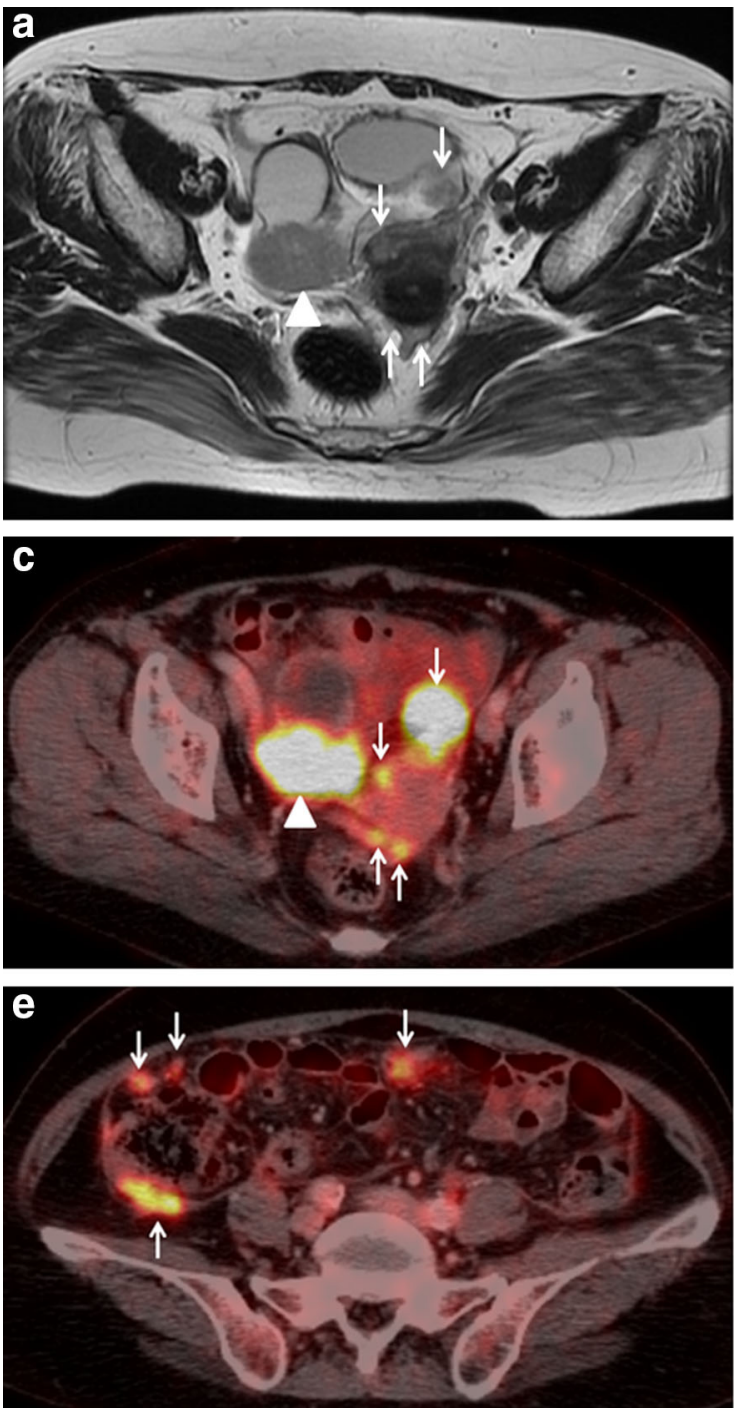

Fig. 1 A 63-year-old woman with primary ovarian cancer with peritoneal dissemination. a T2-weighted MRI before the chemotherapy shows a right ovarian solid mass (arrowhead) and multiple peritoneal disseminations (arrows). b Maximum intensity projection (MIP) of the FDG-PET image shows multiple intense uptake points in the pelvis and abdomen. $\mathbf{c}$ PET/contrast-enhanced CT and $\mathbf{d}$ enhanced $\mathrm{CT}$ portion in the pelvis show abnormal FDG uptake corresponding to

\section{Initial staging}

Accurate staging at the time of diagnosis is extremely important when attempting to plan adequate therapy, and it is the most important prognostic factor. For pretreatment staging of gynecologic malignancies, MRI and CT are considered the most useful imaging techniques for assessing the pelvis and chest/abdomen, respectively. High-resolution MRI with contrast material and diffusion-weighted imaging is probably still the best imaging technique for initial primary tumor staging, such as tumor extension and its relationship with surrounding tissue. Although several reports have indicated the diagnostic accuracy of FDG-
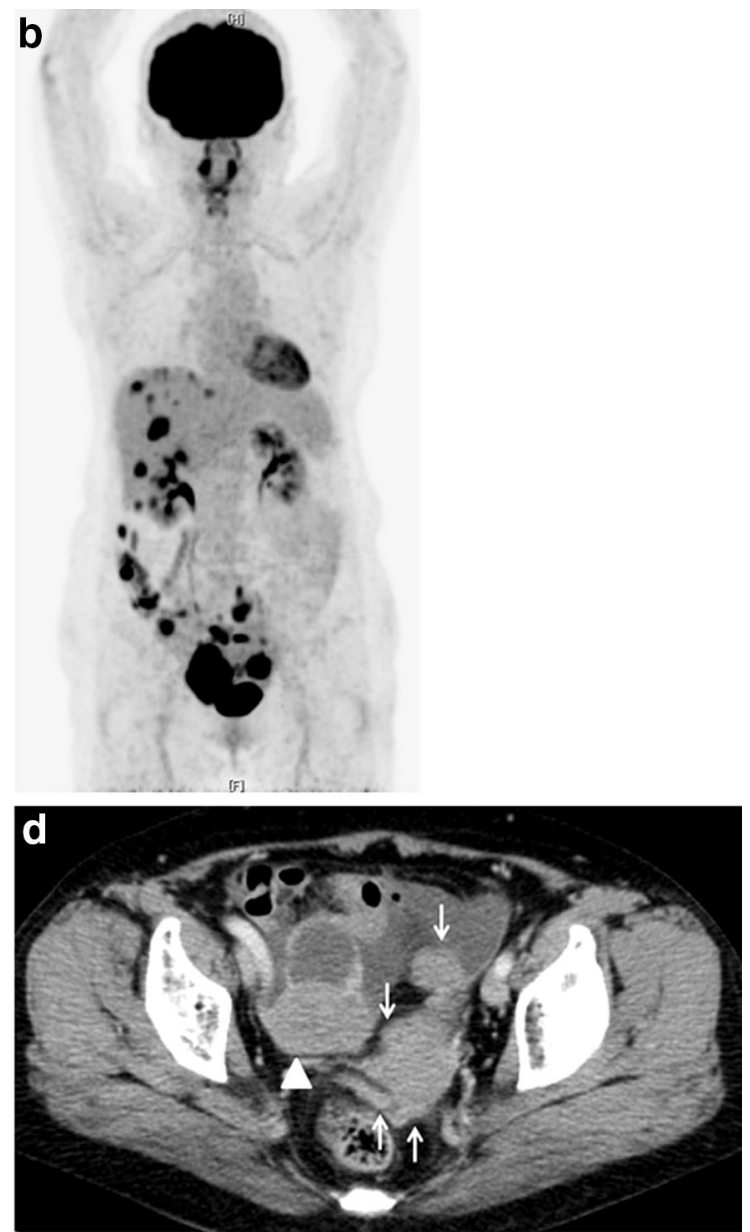

a right ovarian solid tumor (arrowhead) and multiple peritoneal disseminations (arrows). e PET/contrast-enhanced CT shows multiple peritoneal disseminations with intense FDG uptake (arrows). Postchemotherapy surgery revealed viable high-grade serous adenocarcinoma of the right ovary and multiple peritoneal disseminations (ypT3bN0 stage IIIb) 

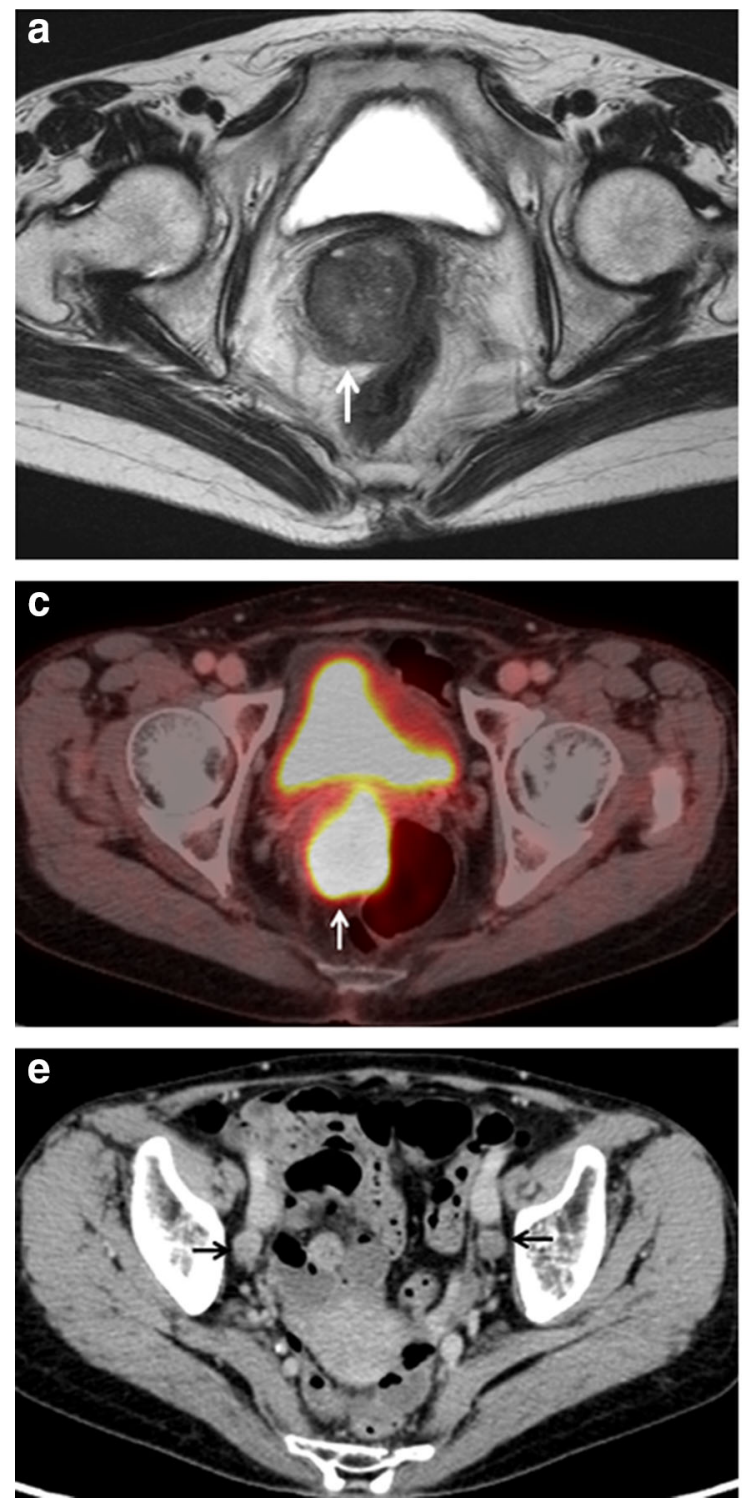

Fig. 2 A 59-year-old woman with uterine cervical cancer with bilateral pelvic lymph node (LN) metastases. a T2-weighted MRI clearly shows a cervical bulky mass with posterior parametrial involvement and no invasion to the bladder or rectum (arrow). b MIP of the FDG-PET image shows multiple intense uptake in the pelvis (arrows) and bilateral hilum. The uptake in the bilateral hilum is judged to be physiological uptake by LNs. c FDG-PET/contrast-

PET/CT for ovarian cancer staging [6-8], its role in staging remains controversial, and its clinical significance is unclear. PET/CECT is an adjunct to conventional staging procedures, and should not be a replacement for MRI.

Generally, the role of FDG-PET/CT in oncology has been to assess lymph node $(\mathrm{N})$ and distant metastasis $(\mathrm{M})$, rather than to determine tumor extension and its relationship with surrounding tissues (T). PET/CT plays an effective role in staging for patients with advanced disease, providing useful information on extrapelvic sites, such as supraclavicular lymph nodes (LNs), para-aortic LNs,
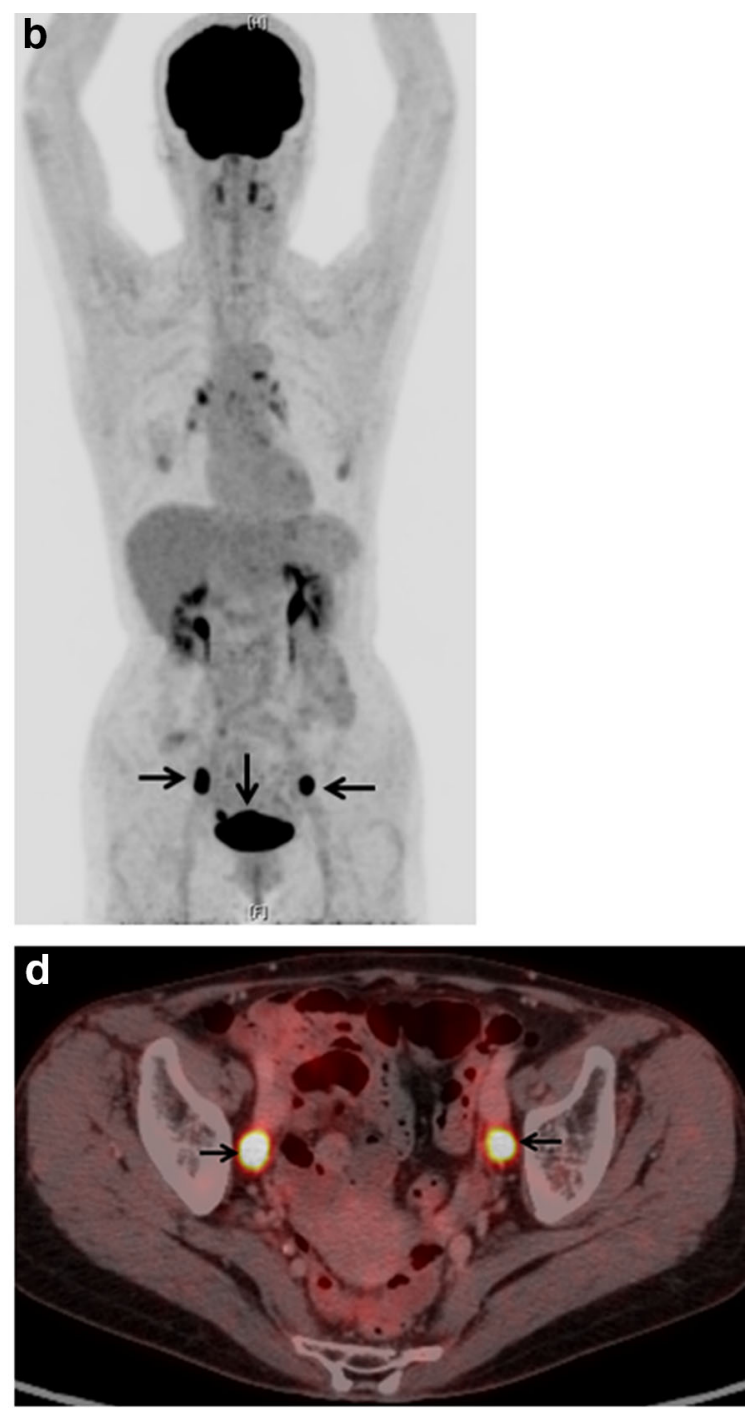

enhanced CT in the pelvis shows a cervical mass with intense FDG uptake (arrow). d PET/contrast-enhanced CT and e enhanced CT show swelling of the bilateral obturator LNs with intense FDG uptake (arrows). Surgery was undertaken, and the pathological findings were cervical squamous cell carcinoma invading the peritoneum and bilateral obturator LNs (pT2bN1c)

peritoneum, omentum, bone, and muscle. However, the added value of PET/CT for the initial staging of early-stage gynecologic cancer without $\mathrm{LN}$ or distant metastases is limited. Figures 1 and 2 show two examples of FDG-PET/ contrast-enhanced CT images obtained for the initial staging of a patient with ovarian cancer invading the peritoneum and a patient with cervical cancer invading pelvic LNs, respectively.

Definition of LN status is one of the most important prognostic factors in patients with gynecologic malignancies, and the survival rates of patients with nodal 
metastases are significantly lower than those of patients without such metastases. Although surgical LN assessment is the reference standard for the diagnosis of LN metastasis, surgical lymphadenectomy increases the risk of immediate and delayed complications, and also the time and cost of diagnosis. If a noninvasive imaging technique were available to accurately identify LN metastases, unnecessary lymphadenectomy procedures could be avoided and the radiation field could be modified for individual cases. Although CT and MRI are widely used to assess LNs, they are not sufficiently accurate for adequate evaluation of nodal spread, and their sensitivity is very low in view of their size criterion (the most accepted of which is a shortaxis diameter exceeding $10 \mathrm{~mm}$ ). FDG-PET is a functional method based on the increased glucose metabolism of cancer cells, regardless of node size, and PET/CT can often detect metastatic LNs $5-9 \mathrm{~mm}$ in size. FDG-PET/CT is superior to conventional imaging techniques, but because of its moderate sensitivity it should not replace lymphadenectomy. PET/CT is unable to diagnose micrometastasis of LNs.

\section{Cervical cancer}

Choi et al. [9] compared the accuracy of FDG-PET/CT and MRI for detecting pelvic and para-aortic LN metastasis in 154 regions (seven regions per patent: the para-aortic area; both common iliac areas; both external areas; and both internal/obturator areas) in 22 patients with FIGOIB through IVA cervical cancer, and demonstrated that PET/ CT was more sensitive than MRI (58 vs. $30 \%$ ), although no significant differences were noted with regard to the specificity (93 vs. $93 \%$ ) and accuracy ( 85 vs. $73 \%$ ) of these methods. Sironi et al. [10] prospectively examined 1081 pelvic LNs in 47 patients with early-stage cervical cancer (FIGO stage IA or IB) and demonstrated that the sensitivity, specificity, and accuracy of overall node-based analysis using FDG-PET/CT were $72 \%$ (13/18), $99 \%$ (1060/1063), and $99 \%(1073 / 1081)$, respectively: the equivalent values from patient-based analysis were $73 \%$ (11/15), $97 \%$ (31/32), and $89 \%$ (42/47), respectively. They also reported that the sensitivity of PET/CT-based diagnosis for LNs with a diameter equal to or lower than $5 \mathrm{~mm}$ and larger than $5 \mathrm{~mm}$ were $0 \%(0 / 5)$ and $100 \%$ (13/13), respectively. A systematic review and meta-analysis including ten studies (385 patients in total) showed that, although the pooled specificity of FDG-PET/CT or PET for the detection of para-aortic LN metastases in patients with cervical cancer was consistent $[97 \%, 95 \%$ confidence interval (CI) 93-99\%], its sensitivity was low and heterogeneous $(34.0 \%, 95 \%$ CI $10-72 \%)$ [11]. In five studies including patients with a high prevalence $(>15 \%)$ of para-aortic LN metastasis (136 patients in total), the estimated sensitivity and specificity were $73 \%$ (95\% CI $53-87 \%)$ and $93 \%$ (95\% CI $86-97 \%)$, respectively.

\section{Endometrial cancer}

A recent systematic review and meta-analysis (243 patients in total) including seven pooled diagnostic indices (per patient basis) for the detection of pelvic and/or para-aortic LN metastases from endometrial cancer by FDG-PET/CT or PET showed that sensitivity was $63 \%(95 \% \mathrm{CI}$ 48.7-75.7\%), specificity $95 \%$ (95\% CI 90.4-97.4\%), and overall diagnostic accuracy $90 \%$ [12].

\section{Ovarian cancer}

In a recent systematic review and meta-analysis (882 patients in total) including eighteen papers for detection of LN metastases in patients with ovarian cancer, FDG-PET/ CT or PET showed the highest pooled sensitivity [73\% (95\% CI 68-78\%)] and specificity [97\% (95\% CI 96-98\%)], while the corresponding figures for CT were $43 \%$ (95\% CI 36-50\%) and $95 \%$ (95\% CI 93-96\%), and those for MRI were $55 \%$ (95\% CI 44-55\%) and $88 \%$ (95\% CI 85-91\%), respectively [13].

\section{Optimization of treatment}

\section{Cervical cancer}

Chao et al. [14] reported a prospective trial in 47 cervical cancer patients with para-aortic, inguinal, and/or supraclavicular nodal metastases evident on CT/MRI. Additional FDG-PET or PET/CT had a positive clinical impact on treatment decision-making in 21 patients (45\%), no impact in 23 , and a negative impact in three. Information with a positive impact included demonstration of additional curable sites $(n=8)$, down-staging $(n=6)$, facilitation of biopsy for involved sites $(n=4)$, or changing the treatment goal to palliation $(n=3)$. Lin et al. [15] demonstrated that information with a negative impact included unnecessary LN (supraclavicular, axillary, and inguinal LN) biopsy due to false-positive PET/CT results, the biopsy samples later proving negative. Combined with cisplatin-based chemotherapy, external beam radiation therapy continues to be the standard therapy for patients with locally advanced cervical cancer. PET/CT plays a meaningful role in external-beam radiotherapy planning, giving a more certain gross target volume and planning target volume and reducing the radiation dose to surrounding normal structures. Moreover, Esthappan et al. [16] have proposed PET/CT-guided intensity-modulated 
radiation therapy for para-aortic LNs in patients with cervical cancer in an attempt to escalate radiation doses with acceptable sparing of surrounding normal structures, particularly the bowel. Belhocine et al. [17] reported that FDG-PET staging significantly influenced treatment decision-making in $18 \%$ of their patients. They concluded that for patients with positive pelvic LNs and negative paraaortic LNs, the benefit of para-aortic LN dissection ought to be assessed before changing the treatment plan, and that PET/CT might be used for biopsy guidance. For patients with neither pelvic nor para-aortic LN involvement, PET may obviate the need for histological confirmation and allow the use of chemoradiation instead of surgery. Patients in whom PET/CT demonstrates para-aortic spread should be offered extended-field treatment. Chao et al. [14] reported the results of a prospective trial that included 47 cervical cancer patients with para-aortic, inguinal, or supraclavicular nodal involvement as found at CT or MRI. Additional FDG PET or PET/CT had a positive clinical effect on the care of 21 patients (44.7\%), including the depiction of additional curable sites, downstaging of disease, enabling metabolic biopsy of involved sites, and changing the treatment strategy to palliation.

\section{Tumor recurrence/restaging}

The detection and precise localization of recurrent lesions is a critical task, as the results guide management and the selection of an appropriate therapeutic approach, thus helping to prolong survival. Tumor markers can be very useful for detecting recurrence. However, the use of tumor markers does not facilitate localization of recurrence, which is very important for treatment planning. Because of its high sensitivity and specificity in comparison with conventional techniques such as CT and MRI, FDG-PET/CT is very useful for detecting recurrent or residual tumors. However, PET/CT may yield false-negative results in patients with small, necrotic, mucinous, cystic, or low-grade tumors. In addition, in the post-therapy setting, inflammatory and infectious processes may lead to false-positive PET/CT results. Despite these drawbacks, $\mathrm{PET} / \mathrm{CT}$ is superior to $\mathrm{CT}$ and MR imaging at depicting recurrent disease. PET/CT can also evaluate the whole body (except the brain) in a single examination and may have the greatest utility in situations where tumor marker levels are rising and conventional imaging studies show negative or equivocal findings. Moreover, PET/CT provides useful information for patient management, because the findings may prompt a change in management involving unplanned treatment, a change in the treatment plan, or obviate the need for planned treatment. Figure 3 shows an example of PET/CT images obtained for the restaging of an endometrial cancer patient with local recurrence, pelvic side wall and obturator LN metastasis, and multiple lung metastases.

\section{Cervical cancer}

A recent systematic review and meta-analysis including eight studies (221 patients in total) found a sensitivity of $94 \%$ (95\% CI 90-97\%) and a specificity of $84 \%(95 \%$ CI 75-91\%) for pooled diagnostic indices (per patient basis) for FDG-PET/CT-based detection of overall recurrence in patients with cervical cancer [18].

\section{Endometrial cancer}

A recent systematic review and meta-analysis including eleven studies (541 patients in total) found a sensitivity of $96 \%$ (95\% CI $92-98 \%)$ and a specificity of $93 \%(95 \%$ CI $89-95 \%$ ) for pooled diagnostic indices (per patient basis) for the detection of overall recurrence in patients with endometrial cancer by FDG-PET/CT or PET. The treatment plan was changed in $22-35 \%$ of the studied patients [19].

\section{Ovarian cancer}

Many reports have discussed the usefulness of FDG-PET/CT for restaging patients with ovarian cancer [20-27]. When the gold standard was clinical follow-up including radiological imaging, the diagnostic accuracy of PET/CT was very high, with 79-97\% sensitivity, 80-97\% specificity, and 85-95\% accuracy in patient-based analysis [23-26]. However, when the gold standard was histopathology after surgery, the diagnostic accuracy of PET/CT tended to be poorer, with corresponding figures of 53-83, 40-86, and 63-82 \%, respectively [24-26]. The discrepancies in these values between clinical follow-up and surgical histopathology as gold-standard assessments may depend partly on the resolution of the PET/ CT system used and partly on the size of microscopically small lesions, from which few positron/gamma rays are emitted. The proportion of cases in which clinical treatment was changed on the basis of PET/CT findings was 25-58 \% $[20,23$, 27]. Among patients with recurrent ovarian cancer, Ebina et al. [27] demonstrated that FDG-PET or PET/CT was useful for selecting candidates for cytoreductive surgery, as such patients tended to be those with a treatment-free interval of $\geq 12$ months to avoid any attempt at surgery for miliary dissemination. In a meta-analysis comparing techniques for detecting ovarian cancer recurrence, Gu et al. [28] found that PET/CT [sensitivity: $91 \%$ (95 \% CI 88-94 \%); specificity: $88 \%$ (95\% CI 81-93\%)] performed better than CT [sensitivity: $79 \%$ (95\% CI 74-84 \%); specificity: $84 \%$ (95\% CI 76-90 \%)] or MRI [sensitivity: $75 \%$ (95\% CI 69-80\%); specificity: $78 \%$ (95\% CI 70-85\%)]. 

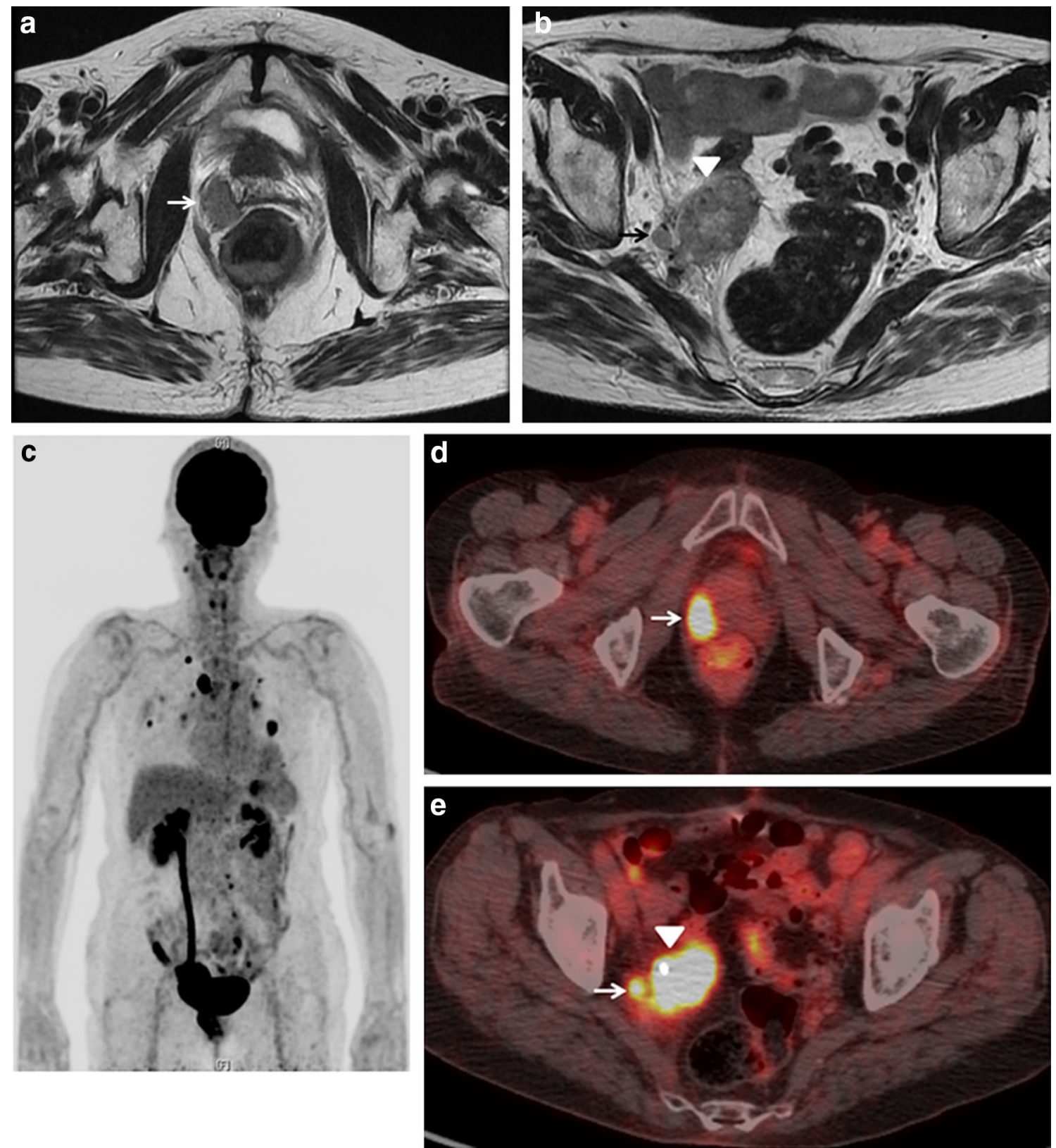

Fig. 3 A 79-year-old woman with recurrent lesions consisting of local recurrence, right pelvic side wall invasion, obturator and paraaortic LN metastasis, and multiple lung metastases after surgery for endometrial cancer. a T2-weighted MRI clearly shows a solid mass at the right vaginal stump (arrow), suggesting local recurrence. b T2weighted MRI clearly shows a bulky mass in the right pelvic side wall (arrowhead), suggesting pelvic side wall recurrence and a tiny right obturator LN (arrow). c MIP of the FDG-PET image shows multiple

\section{Treatment monitoring}

At present, conventional anatomical imaging is commonly used to evaluate response to treatment by assessing changes in tumor size. However, CT and MRI have limited value for detecting responses early after the initiation of therapy, because it takes time for an anatomic response to intense uptake in the pelvis, abdomen, and chest. Right hydronephrosis is evident. d PET/CT shows abnormal FDG uptake corresponding to the right vaginal stump (arrow), confirming the presence of local recurrence. e PET/CT shows abnormal FDG uptake corresponding to the right pelvic side wall (arrowhead) and a tiny right obturator LN (arrow), confirming the presence of pelvic-side wall and obturator $\mathrm{LN}$ recurrences

become noticeable. Metabolic changes often precede morphological changes in tumor response, and therefore FDG-PET is able to demonstrate a response sooner than CT [29]. FDG-PET is thought to be useful for picking up nonresponders to chemotherapy at an earlier stage. Identifying nonresponders would significantly improve patient management by reducing the use of ineffective therapies, 
preventing adverse effects, reducing any delay before the administration of more effective treatment, and minimizing costs.

\section{Uterine and ovarian cancer}

Nishiyama et al. [30] demonstrated that FDG-PET-derived parameters, including the maximum standardized uptake value (SUVmax) and percentage change, had the potential to predict response to chemotherapy or chemoradiotherapy in 21 patients with advanced gynecologic cancer (uterine cervical cancer, $n=9$; endometrial cancer, $n=4$; ovarian cancer, $n=8$ ). Based on histopathologic analysis of the specimens obtained at surgery, 10 patients were found to be responders and 11 nonresponders. When an arbitrary SUVmax of 3.8 was taken as the cutoff for differentiating between responders and nonresponders after therapy, FDG-PET showed a sensitivity of $90 \%$, a specificity of $63.6 \%$, and an accuracy of $76.2 \%$. When an arbitrary percentage change of $65 \%$ was taken as the cutoff, FDG-PET showed a sensitivity of $90 \%$, a specificity of $81.8 \%$, and an accuracy of $85.7 \%$.

\section{Cervical cancer}

Grigsby et al. [31] retrospectively compared metabolic response to therapy with outcome in 152 patients with cervical cancer. They compared the results of PET performed before and after chemoradiation therapy 1-12 months (mean: 3 months) after completion of treatment. The 5-year causespecific survival rates were $80 \%$ for 114 patients with abnormal FDG uptake (complete metabolic response), $32 \%$ for 20 patients with persistent uptake (partial metabolic response), and $0 \%$ for 18 patients with new uptake (progressive disease) at the post-treatment examination $(p<0.001)$. Schwarz et al. [32] prospectively demonstrated that 3-month post-therapy FDG uptake was predictive of survival. They compared the PET results obtained before and after therapy (external irradiation, brachytherapy, and concurrent chemotherapy) performed 2-4 months (mean 3 months) after completion of treatment in 92 patients with cervical cancer. The 3-year progression-free survival rates were $78 \%$ for 65 patients with a complete metabolic response, $33 \%$ for 15 patients with a partial metabolic response, and $0 \%$ for 12 patients with new uptake at the posttreatment examination $(p<0.001)$. These studies demonstrate that FDG-PET can provide reliable, long-term prognostic information only 3 months after the completion of therapy.

\section{Ovarian cancer}

Avril et al. [33] demonstrated that FDG-PET may be a promising technique for early prediction of the response to chemotherapy and for predicting the response after the completion of chemotherapy. They demonstrated a significant correlation between changes in tumor tracer after the first and third cycles of chemotherapy, but not with conventional clinical or CA-125 response criteria. A higher rate of complete tumor resections was achieved in metabolic responders (defined as $>20 \%$ reduction in SUV after the first cycle and $>50 \%$ after the third cycle) than in nonresponders, and macroscopically tumor-free surgery was achieved in $33 \%$ of metabolic responders, compared with only $13 \%$ of nonresponders. Metabolic responders had a longer median overall survival than did nonresponders. By using a threshold for the decrease in SUV from the baseline of $20 \%$ after the first cycle, median overall survival was found to be 38.3 months in metabolic responders, compared with 23.1 months in metabolic nonresponders. Using a $55 \%$ decrease in SUV after the third cycle as the threshold, median overall survival was 38.9 months in metabolic responders, whereas it was 19.7 months in nonresponders. Kim et al. [34] compared the prognosis of 55 patients evaluated by either FDG-PET or second-look laparotomy after cytoreductive surgery and adjuvant chemotherapy. PET had a prognostic value similar to that of second-look surgery. There was no significant difference in progression-free interval (28.8 vs. 30.6 months) or disease-free interval in the PET-negative group (40.5 vs. 48.6 months). They suggested that FDGPET can be used to replace second-look laparotomy, although further investigations of larger groups of patients will be needed.

\section{Pretreatment prognostic prediction}

The degree of FDG uptake correlates with tumor proliferation rates, reflecting tumor aggressiveness. Several authors have demonstrated that FDG uptake by a primary gynecologic tumor and LNs may also be correlated with patient outcome.

\section{Cervical cancer}

Kidd et al. [35] evaluated the pre-treatment SUVmax derived from FDG-PET imaging of the primary tumor and its association with treatment response and prognosis in 287 patients with stage I A2 through IVB cervical cancer. Among tumor histology, tumor volume, LN metastasis, and SUVmax, a Cox proportional hazards model for death showed that SUVmax was the only significant independent factor. The overall 5-year survival rates were $95 \%$ for SUVmax $<5.2,70 \%$ for SUVmax 5.2-13.3, and $44 \%$ for SUVmax $>13.3(p<0.0001)$. The same group also demonstrated that nodal involvement detected by FDG-PET or 
PET/CT in a large cohort of 560 patients with I A2 through IVA cervical cancer was related to clinical stage, comparable to historical data, and able to stratify patient recurrence and survival outcome [36].

\section{Endometrial cancer}

Nakamura et al. [37] investigated the prognostic value of preoperative SUVmax derived from FDG-PET/CT imaging of the primary tumor in 131 patients with stage IA through IVB endometrial cancer. They found that SUVmax was significantly correlated with FIGO stage, histologic grade, depth of myometrial invasion, lymph node metastasis, lymphovascular space involvement, and maximum tumor size. Multivariate analysis showed that SUVmax and FIGO stage were independent prognostic factors for both diseasefree survival and overall survival.

\section{Ovarian cancer}

Risum et al. [38] investigated the role of FDG-PET/CT for evaluating predictors of overall survival in 152 patients with stage IIIC/IV ovarian cancer, and for selecting patients for neoadjuvant chemotherapy. Univariate analysis showed that incomplete debulking, pleural exudates, postmenopausal status, WHO performance status greater than 2, PET/CT stage IV, and large bowel mesentery implantation were statistically significant prognostic variables, and multivariate Cox regression analysis showed that incomplete debulking was the only significant independent prognostic variable. They also suggested that PET/CT criteria for the referral of patients with advanced ovarian cancer to neoadjuvant chemotherapy were PET/CT stage IV, pleural exudates, and PET-positive large bowel mesentery implantation.

\section{Pitfalls or limitation}

FDG is physiologically accumulated in the bowel and excreted through the urinary tract. This might interfere with the optimal evaluation of primary tumors in the abdomen and pelvis. Urinary bladder activity can be avoided by asking the patient to empty her urinary bladder before the examination. Other approaches include the use of a urinary catheter for continuous drainage, or hydration with $1,000 \mathrm{ml}$ of intravenous normal saline along with diuretics such as furosemide [39]. Moreover, in the abdomen and pelvis, a large number of misregistrations can occur as a result of physiologic uptake in the bowel and bladder, and as a result of bowel peristalsis; knowledge of these issues and careful review are required for accurate interpretation.
Some pitfalls in interpreting FDG-PET/CT also need to be considered, such as accumulation of FDG in the normal uterus, ovary, and activated inflammatory cells. A physiological increase of endometrial FDG activity may also occur during the menstrual and ovulatory phases of the menstrual cycle [40]. Increased physiological ovarian FDG accumulation may also be evident around the time of ovulation and during the early luteal phase of the menstrual cycle in premenopausal woman [41].

FDG-PET/CT is limited in its ability to identify lesions measuring $<1 \mathrm{~cm}$, and in particular those smaller than $5 \mathrm{~mm}$. FDG-PET/CT can only detect lesions containing a volume of malignant cells that is sufficient to change the observed glucose metabolism, and neither of these imaging modalities can detect tiny lesions. This inadequate performance of PET and PET/CT is not surprising, because $5 \mathrm{~mm}$ corresponds to the mean spatial resolution of the PET components, i.e., within the range $4-6 \mathrm{~mm}$. This limited spatial resolution of the PET component makes the presence of metastasis in small LNs barely detectable. Among malignant neoplasms, predominantly cystic, necrotic, or mucinous tumors may exhibit poor accumulation of FDG. Necrotic LNs within the pelvis or abdomen may also show little or no FDG activity. In these situations, the information provided by the CT images (preferably optimized full-dose diagnostic CT, discussed later) of the PET/CT study can be extremely valuable. These problems can be resolved by PET/MRI, as mentioned later.

\section{PET/contrast-enhanced CT}

In recent years, PET/contrast-enhanced CT with intravenous iodine contrast medium and a sufficient radiation dose has been gradually introduced clinically. The use of contrast enhancement would be expected to increase diagnostic confidence, as differentiation between adjacent softtissue structures and accurate localization and extension of tumors is often difficult with unenhanced CT images. If there is a high index of suspicion from PET in the pelvis, contrast-enhanced and high-dose CT of inline PET/CT can accurately differentiate pathological uptake of FDG by LNs from physiological uptake by vessels, bowel, or ureter in comparison with unenhanced and low-dose CT. In fact, many of these fused images are used to diagnose various malignant tumors including gynecologic cancer in our institutions [6, 23, 42]. Although the latest CT-based attenuation correction algorithms reduce overestimation by de-emphasizing high-density areas due to contrast medium, without incurring significant errors in quantification [43], unenhanced and low-dose CT is performed for attenuation correction in many centers, including our institution. The International Atomic Energy Agency (IAEA) demonstrated 
that when a contrast-enhanced diagnostic $\mathrm{CT}$ is justified for clinical reasons, it should, where possible, also be used to avoid an additional low-dose scan [44]. An example of a PET/CT examination protocol consisting of a topogram (0.2-0.8 mSv), a low-dose CT scan for attenuation correction (e.g., $130 \mathrm{kVp}, 40 \mathrm{mAs}, 1-4 \mathrm{mSv}$ ), an FDG-PET scan $(5.7-7.0 \mathrm{mSv})$, and a diagnostic $\mathrm{CT}$ scan acquired after the administration of an intravenous CT contrast medium $(130 \mathrm{kVp}, 160 \mathrm{mAs}, 14-19 \mathrm{mSv})$ with a total dose of $25 \mathrm{mSv}$ is shown. After a routine PET/CT study, we perform a diagnostic CT study with iodine medium immediately, keeping the patient in the same position.

\section{Other FDG traces}

Torizuka et al. [45] compared FDG-PET with ${ }^{11} \mathrm{C}$-choline PET in a population of 21 patients (18 untreated) with gynecological malignancies and three with a suspected relapse, and found that ${ }^{11} \mathrm{C}$-choline PET detected lesions in a higher number of patients than did FDG-PET.

Tsujikawa et al. [46] compared $16 \alpha-\left[{ }^{18}\right.$ F]fluoro-17 $\beta$ estradiol (FES) and FDG-PET in 38 patients with benign and malignant uterine tumors to compare differences in tracer accumulation. FES uptake seems higher in benign lesions, while malignancies are more FDG-avid, and vice versa. The same group evaluated the tumor aggressiveness of endometrial cancer using FES and FDG, and reported that high-risk carcinoma (FIGO $\geq$ Ic or histologic grade $\geq 2$ ) showed a significantly greater FDG-to-FES ratio $(3.6 \pm 2.1)$ than did low-risk carcinoma (FIGO $\leq \mathrm{Ib}$ and histological grade $1 ; 1.3 \pm 0.5, p<0.01$ ) [47]. In a study of 38 patients with cervical cancer who had undergone chemoradiotherapy, Dehdashti et al. [48] showed that pretreatment tumor hypoxia demonstrated by PET with ${ }^{60} \mathrm{Cu}$-labeled diacetylbis $\left(N^{4}\right.$-methylthiosemicarbazone $)$ $\left({ }^{60} \mathrm{Cu}\right.$-ATSM) was a biomarker of poor prognosis (progression-free survival and cause-specific survival).

\section{Integrated PET/MRI}

In recent years, there has been increasing interest in the development of integrated PET/MRI systems, following the success of hybrid imaging with PET/CT. PET/MRI would have a number of advantages, such as improved soft-tissue contrast, the possibility of performing truly simultaneous instead of sequential acquisitions, and the availability of sophisticated MRI sequences, such as diffusion and perfusion imaging, functional MRI, and MR spectroscopy, which can add important information. Moreover, the use of PET/MRI would result in a significant decrease in radiation exposure, which is of foremost importance for serial follow-up and pediatric imaging.

A combined PET/MRI scanner would provide an alternative to a combined PET/CT scanner for whole-body oncologic imaging [49-52]; improved accuracy could be achieved in the detection, staging, characterization, and functional therapy monitoring of several types of cancer. However, prototype designs for human whole-body PET/ MRI systems still have a number of technical and methodological difficulties that need to be overcome: these include the compatibility of both the PET and MRI components in an integrated system, and merging the two technologies, whose signals are prone to distortion.

\section{Conclusion}

FDG-PET/CT can allow combined metabolic and morphological assessment of tumors with significant improvements in diagnostic accuracy, resulting in a considerable impact on patient management, diagnosis, staging, treatment optimization, re-staging, therapy monitoring, and prognostication of gynecological cancer. In the future, tracers other than FDG and integrated PET/MRI will be realized.

Conflict of interest We declare no financial support or relationship that may pose a conflict of interest.

\section{References}

1. Risum S, Hogdall C, Loft A, Berthelsen AK, Høgdall E, Nedergaard $\mathrm{L}$, et al. The diagnostic value of PET/CT for primary ovarian cancer: a prospective study. Gynecol Oncol. 2007;105:145-9.

2. Castelluci P, Perrone AM, Picchio M, Ghi T, Farsad M, Nanni C, et al. Diagnostic accuracy of ${ }^{18} \mathrm{~F}$-FDG PET/CT in characterizing ovarian lesions and staging ovarian cancer: correlation with transvaginal ultrasonography, computed tomography, and histology. Nucl Med Commun. 2007;28:589-95.

3. Nam EJ, Yun MJ, Oh YT, Kim JW, Kim JH, Kim S, et al. Diagnosis and staging of primary ovarian cancer: correlation between PET/CT, Doppler US, and CT or MRI. Gynecol Oncol. 2010;116:389-94.

4. Kitajima K, Suzuki K, Senda M, Kita M, Nakamoto Y, Onishi Y, et al. FDG-PET/CT for diagnosis of primary ovarian cancer. Nucl Med Commun. 2011;32:549-53.

5. Tanizaki Y, Kobayashi A, Shiro M, Ota N, Takano R, Mabuchi Y, et al. Diagnostic value of preoperative SUVmax on FDG-PET/ $\mathrm{CT}$ for the detection of ovarian cancer. Int $\mathrm{J}$ Gynecol Cancer. 2014;24:454-60.

6. Kitajima K, Murakami K, Yamasaki E, Kaji Y, Fukasawa I, Inaba $\mathrm{N}$, et al. Diagnostic accuracy of integrated FDG-PET/contrastenhanced $\mathrm{CT}$ in staging ovarian cancer: comparison with enhanced CT. Eur J Nucl Med Mol Imaging. 2008;35:1912-20.

7. De Iaco P, Musto A, Orazi L, Zamagni C, Rosati M, Allegri V, et al. FDG-PET/CT in advanced ovarian cancer staging: value and pitfalls in detecting lesions in different abdominal and pelvic 
quadrants compared with laparoscopy. Eur J Radiol. 2011;80: 98-103.

8. Hynninen J, Kemppainen J, Lavonius M, Virtanen J, Matomäki J, Oksa S, et al. A prospective comparison of integrated FDG-PET/ contrast-enhanced CT and contrast-enhanced CT for pretreatment imaging of advanced epithelial ovarian cancer. Gynecol Oncol. 2013;131:389-94.

9. Choi HJ, Roh JW, Seo SS, Lee S, Kim JY, Kim SK, et al. Comparison of the accuracy of magnetic resonance imaging and positron/emission tomography in the presurgical detection of lymph node metastases in patients with uterine cervical carcinoma. Cancer. 2006;106:914-22.

10. Sironi S, Buda A, Picchio M, Perego P, Moreni R, Pellegrino A, et al. Lymph node metastasis in patients with clinical early-stage cervical cancer: detection with integrated FDG PET/CT. Radiology. 2006;238:272-9.

11. Kang S, Kim SK, Chung DC, Seo SS, Kim JY, Nam BH, et al. Diagnostic value of ${ }^{18} \mathrm{~F}$-FDG PET for evaluation of paraaortic nodal metastasis in patients with cervical carcinoma: a metaanalysis. J Nucl Med. 2010;51:360-7.

12. Chang MC, Chen JH, Liang JA, Yang KT, Cheng KY, Kao CH. ${ }^{18}$ F-FDG PET or PET/CT for detection of metastatic lymph nodes in patients with endometrial cancer: a systematic review and meta-analysis. Eur J Radiol. 2012;81:3511-7.

13. Yuan Y, Gu ZX, Tao XF, Liu SY. Computer tomography, magnetic resonance imaging, and positron emission tomography or positron emission tomography/computer tomography for detection of metastatic lymph nodes in patients with ovarian cancer: a meta-analysis. Eur J Radiol. 2012;81:1002-6.

14. Chao A, Ho KC, Wang CC, Cheng HH, Lin G, Yen TC, et al. Positron emission tomography in evaluating the feasibility of curative intent in cervical cancer patients with limited distant lymph node metastases. Gynecol Oncol. 2008;110:172-8.

15. Lin LL, Mutic S, Low DA, LaForest R, Vicic M, Zoberi I, et al. Adaptive brachytherapy treatment planning for cervical cancer using FDG-PET. Int J Radiat Oncol Biol Phys. 2007;67:91-6.

16. Esthappan J, Chaudhari S, Santanam L, Mutic S, Olsen J, Macdonald DM, et al. Prospective clinical trial of positron emission tomography/computed tomography image-guided intensity-modulated radiation therapy for cervical carcinoma with positive para-aortic lymph nodes. Int J Radiat Oncol Biol Phys. 2008;72: 1134-9.

17. Belhocine T, Thille A, Fridman V, Albert A, Seidel L, Nickers P, et al. Contribution of whole-body 18FDG PET imaging in the management of cervical cancer. Gynecol Oncol. 2002;87:90-7.

18. Chu Y, Zheng A, Wang F, Lin W, Yang X, Han L, et al. Diagnostic value of ${ }^{18}$ F-FDG-PET of PET-CT/in recurrent cervical cancer: a systematic review and meta-analysis. Nucl Med Commun. 2014;35:144-50.

19. Kadkhodayan S, Shahriari S, Treglia G, Yousefi Z, Sadeghi R. Accuracy of 18-F-FDG PET imaging in the follow up of endometrial cancer patients: systematic review and meta-analysis of the literature. Gynecol Oncol. 2013;128:397-404.

20. Chung HH, Kang WJ, Kim JW, Park NH, Song YS, Chung JK, et al. Role of $\left[{ }^{18} \mathrm{~F}\right]$ FDG PET/CT in the assessment of suspected recurrent ovarian cancer: correlation with clinical or histological findings. Eur J Nucl Med Mol Imaging. 2007;34:480-6.

21. Sebastian S, Lee SI, Horowitz NS, Scott JA, Fischman AJ, Simeone JF, et al. PET-CT vs. CT alone in ovarian cancer recurrence. Abdom Imaging. 2008;33:112-8.

22. Iagaru AH, Mittra ES, McDougall IR, Quon A, Gambhir SS. ${ }^{18} \mathrm{~F}-$ FDG PET/CT evaluation of patients with ovarian carcinoma. Nucl Med Commun. 2008;29:1046-51.

23. Kitajima K, Murakami K, Yamasaki E, Domeki Y, Kaji Y, Fukasawa I, et al. Performance of integrated FDG-PET/contrast enhanced $\mathrm{CT}$ in the diagnosis of recurrent ovarian cancer: comparison with integrated FDG-PET/non-contrast-enhanced CT and enhanced CT. Eur J Nucl Med Mol Imaging. 2008;35: 1439-48.

24. Bristow RE, Del Carmen MG, Pannu HK, Cohade C, Zahurak ML, Fishman EK, et al. Clinically occult recurrent ovarian cancer: patient selection for secondary cytoreductive surgery using combined PET/CT. Gynecol Oncol. 2003;90:519-28.

25. Sironi S, Messa C, Mangili G, Zangheri B, Aletti G, Garavaglia $\mathrm{E}$, et al. Integrated FDG-PET/CT in patients with persistent ovarian cancer: correlation with histologic findings. Radiology. 2004;233:433-40.

26. Pannu HK, Cohade C, Bristow RE, Fishman EK, Wahl RL. PETCT detection of abdominal recurrence of ovarian cancer: radiologicsurgical correlation. Abdom Imaging. 2004;39:398-403.

27. Ebina Y, Watari H, Kaneuchi M, Takeda M, Hosaka M, Kudo M, et al. Impact of FDG PET in optimizing patient selection for cytoreductive surgery in recurrent ovarian cancer. Eur $\mathrm{J}$ Nucl Med Mol Imaging. 2014;41:446-51.

28. Gu P, Pan LL, Wu SQ, Sun L, Huang G. CA125, PET alone, PET-CT, CT and MRI in diagnosing recurrent ovarian carcinoma: a systematic review and meta-analysis. Eur J Radiol. 2009;71:164-74.

29. Dolezelova H, Slampa P, Ondrova B, Gombosova J, Sovadinova S, Novotny T, et al. The impact of PET with ${ }^{18}$ FDG in radiotherapy treatment planning and in the prediction in patients with cervix carcinoma: results of pilot study. Neoplasma. 2008;55:437-41.

30. Nishiyama Y, Yamamoto Y, Kaneishi K, Gombosova J, Sovadinova S, Novotny T, et al. Monitoring the neoadjuvant therapy response in gynecological cancer patients using FDG PET. Eur J Nucl Med Mol Imaging. 2008;35:287-95.

31. Grigsby PW, Siegel BA, Dehdashti F, Rader J, Zoberi I. Posttherapy $\left[{ }^{18} \mathrm{~F}\right]$ fluorodeoxyglucose positron emission tomography in carcinoma of the cervix: response and outcome. J Clin Oncol. 2004;22:2167-71.

32. Schwarz JK, Siegel BA, Dehdashti F, Grigsby PW. Association of post therapy positron emission tomography with tumor response and survival in cervical carcinoma. JAMA. 2007;298: 2289-95.

33. Avril N, Sassen S, Schmalfeldt B, Naehrig J, Rutke S, Weber WA, et al. Prediction of response to neoadjuvant chemotherapy by sequential F-18-fluorodeoxyglucose positron emission tomography in patients with advanced-stage ovarian cancer. J Clin Oncol. 2005;23:7445-53.

34. Kim S, Chung JK, Kang SB, Kim MH, Jeong JM, Lee DS, et al. $\left[{ }^{18} \mathrm{~F}\right]$ FDG PET as a substitute for second-look laparotomy in patients with advanced ovarian carcinoma. Eur J Nucl Med Mol Imaging. 2004;31:196-201.

35. Kidd EA, Siegel BA, Dehdashti F, Grigsby PW. The standardized uptake value for F-18 fluorodeoxyglucose is a sensitive predictive biomarker for cervical cancer treatment response and survival. Cancer. 2007;110:1738-44.

36. Kidd EA, Siegel BA, Dehdashti F, Rader JS, Mutch DG, Powell MA, et al. Lymph node staging by positron emission tomography in cervical cancer: relationship to prognosis. J Clin Oncol. 2010;28:2108-13.

37. Nakamura K, Joja I, Fukushima C, Haruma T, Hayashi C, Kusumoto $\mathrm{T}$, et al. The preoperative SUVmax is superior to ADCmin of the primary tumour as a predictor of disease recurrence and survival in patients with endometrial cancer. Eur J Nucl Med Mol Imaging. 2013;40:52-60.

38. Risum S, Loft A, Engelholm SA, Høgdall E, Berthelsen AK, Nedergaard L, et al. Positron emission tomography/computed tomography predictors of overall survival in stage IIIC/IV ovarian cancer. Int J Gynecol Cancer. 2012;22:1163-9.

39. Koyama K, Okamura T, Kawabe J, Ozawa N, Torii K, Umesaki $\mathrm{N}$, et al. Evaluation of ${ }^{18}$ FDG PET with bladder irrigation in 
patients with uterine and ovarian tumors. J Nucl Med. 2003;44: 353-8.

40. Nishizawa S, Inubushi $\mathrm{M}$, Okada $\mathrm{H}$. Physiological ${ }^{18} \mathrm{~F}-\mathrm{FDG}$ uptake in the ovaries and uterus of healthy female volunteers. Eur J Nucl Med Mol Imaging. 2005;32:549-56.

41. Kim SK, Kang KW, Roh JW, Sim JS, Lee ES, Park SY. Incidental ovarian ${ }^{18} \mathrm{~F}-\mathrm{FDG}$ accumulation on PET: correlation with the menstrual cycle. Eur J Nucl Med Mol Imaging. 2005;32: 757-63.

42. Kitajima K, Suzuki K, Nakamoto Y, Onishi Y, Sakamoto S, Senda $\mathrm{M}$, et al. Low-dose non-enhanced CT versus full-dose contrast-enhanced CT in integrated PET/CT studies for the diagnosis of uterine cancer recurrence. Eur J Nucl Med Mol Imaging. 2010;37:1490-8.

43. Bunyaviroch T, Turkington TG, Wong TZ, Wilson JW, Colsher JG, Coleman RE. Quantitative effects of contrast enhanced CT attenuation correction on PET SUV measurements. Mol Imaging Biol. 2008;10:107-13.

44. IAEA. Radiation protection in newer medical imaging techniques: PET/CT (Safety Reports Series no. 58). http://www-pub. iaea.org/MTCD/publications/PDF/pub1343_web.pdf.

45. Torizuka T, Kanno T, Futatsubashi M, Okada H, Yoshikawa E, Nakamura F, et al. Imaging of gynaecologic tumor: comparison of ${ }^{11}$ C-Choline PET with ${ }^{18}$ F-FDG PET. J Nucl Med. 2003;44: 1051-6.

46. Tsujikawa T, Yoshida Y, Mori T, Kurokawa T, Fujibayashi Y, Kotsuji F, et al. Uterine tumors: pathophysiologic imaging with
$16 \alpha-\left[{ }^{18} \mathrm{~F}\right]$ fluoro-17 $\beta$-estradiol and ${ }^{18} \mathrm{~F}$-fluorodeoxyglucose PETinitial experience. Radiology. 2008;248:599-605.

47. Tsujikawa T, Yoshida Y, Kudo T, Kiyono Y, Kurokawa T, Kobayashi $\mathrm{M}$, et al. Functional images reflect aggressiveness of endometrial carcinoma: estrogen receptor expression combined with ${ }^{18}$ F-FDG PET. J Nucl Med. 2009;50:1598-604.

48. Dehdashti F, Grigsby PW, Lewis JS, Laforest R, Siegel BA, Welch MJ. Assessing tumor hypoxia in cervical cancer by PET with ${ }^{60} \mathrm{Cu}$-labeled diacetyl-bis $\left(N^{4}\right.$-methylthiosemicarbazone). J Nucl Med. 2008;49:201-5.

49. Catalano OA, Rosen BR, Sahani DV, Hahn PF, Guimaraes AR, Vangel MG, et al. Clinical impact of PET/MR imaging in patients with cancer undergoing same-day PET/CT: initial experience in 134 patients - a hypothesis-generating exploratory study. Radiology. 2013;269:857-69.

50. Wetter A, Lipponer C, Nensa F, Heusch P, Rübben H, Altenbernd JC, et al. Evaluation of the PET component of simultaneous $\left[{ }^{18} \mathrm{~F}\right]$ choline PET/MRI in prostate cancer: comparison with $\left[{ }^{18} \mathrm{~F}\right]$ choline PET/CT. Eur J Nucl Med Mol Imaging. 2014;41: 79-88.

51. Kuhn FP, Hüllner M, Mader CE, Kastrinidis N, Huber GF, von Schulthess GK, et al. Contrast-enhanced PET/MR imaging versus contrast-enhanced PET/CT in head and neck cancer: how much MR information is needed? J Nucl Med. 2014;55(4):551-8.

52. Tian J, Fu L, Yin D, Zhang J, Chen Y, An N, Xu B. Does the novel integrated PET/MRI offer the same diagnostic performance as PET/CT for oncological indications? PLoS One. 2014;9:e90844. 